\title{
Contributing authors
}

\author{
Elizabeth Bagshaw \\ School of Earth and Environmental Sciences \\ Cardiff University \\ Main Building, Park Place \\ CF10 3AT Cardiff, UK \\ bagshawe@cardiff.ac.uk
}

James A. Bradley

School of Geography

Queen Mary University of London

London, E1 4NS, United Kingdom

AND

GFZ German Research Centre for Geosciences, Helmholtz Centre Potsdam

Section Interface Geochemistry

Telegrafenberg

14473 Potsdam, Germany

jbradley.earth@gmail.com

\section{Sophie Crevecoeur}

Canada Centre for Inland Waters, Water Science and Technology Branch, Watershed Hydrology and Ecology Research Division,

Environment and Climate Change Canada, Burlington, Ontario, Canada

sophie.crevecoeur@canada.ca

\section{Beat Frey}

Swiss Federal Research Institute WSL

Zürcherstrasse 111

8903 Birmensdorf, Switzerland

beat.frey@wsl.ch

\section{Stefanie A. Hetz}

Institute of Microbiology,

Leibniz University Hannover

Herrenhäuser Strasse 2

30419 Hannover, Germany

stefanie.hetz@ifmb.uni-hannover.de
Marcus A. Horn

Institute of Microbiology, Leibniz

University Hannover

Herrenhäuser Strasse 2

30419 Hannover, Germany

horn@ifmb.uni-hannover.de

\section{Christian Knoblauch}

Institute of Soil Sciences, Universität Hamburg

Allende Platz 2

20146 Hamburg, Germany

Christian.Knoblauch@uni-hamburg.de

\section{Anastasia Komolova}

Institute of Physicochemical and Biological

Problems in Soil Science, Russian Academy

of Sciences

142290 Pushchino, Moscow Region,

Russian Federation

callistephus1@gmail.com

\section{Manoj Kumar}

Natural Resources Institute Finland

Eteläranta 55

96300 Rovaniemi, Finland

manoj.kumar@luke.fi

\section{Susanne Liebner}

GFZ German Research Centre for Geosciences, Helmholtz Centre Potsdam

Section Geomicrobiology

Telegrafenberg

14473 Potsdam, Germany

susanne.liebner@gfz-potsdam.de

\section{Connie Lovejoy}

Département de Biologie, Takuvik Joint International Laboratory, Institut de Biologie Intégrative et des Systèmes (IBIS) and QuébecOcéan, Université Laval, Québec, Canada connie.lovejoy@bio.ulaval.ca 


\section{Stefanie Lutz}

GFZ German Research Centre for Geosciences, Helmholtz Centre Potsdam

Section Interface Geochemistry

Telegrafenberg

14473 Potsdam, Germany

stefanie.lutz@gfz-potsdam.de

\section{Rachel Mackelprang}

California State University Northridge

18111 Nordoff St.

91330 Northridge, CA, USA

rachel.mackelprang@gmail.com

\section{Rhiannon Mondav}

Uppsala University, Department of Ecology and Genetics, Evolutionsbiologiskt Centrum (EBC)

Norbyvägen 18 D

75236 Uppsala, Sweden

rhiannon.mondav@ebc.uu.se

\section{Riitta Nissinen}

University of Jyväskylä, Department of Biological and Environmental Science

P.O. Box 35

40014 Jyväskylä, Finland

riitta.m.nissinen@jyu.fi

\section{Ksenia Novototskaya-Vlasova}

Institute of Physicochemical and Biological Problems in Soil Science, Russian Academy of Sciences

142290 Pushchino, Moscow Region,

Russian Federation

nksusha@gmail.com

\section{Lada Petrovskaya}

Shemyakin \& Ovchinnikov Institute of Bioorganic Chemistry, Russian Academy of Sciences

ul. Miklukho-Maklaya 16/10

117997 Moscow, Russian Federation

Ipetr65@yahoo.com

\section{Ewa Poniecka}

School of Earth and Environmental Sciences

Cardiff University

Main Building, Park Place

CF10 3AT Cardiff, UK

poniecka.ewa@gmail.com

\section{Elizaveta Rivkina}

Institute of Physicochemical and Biological

Problems in Soil Science, Russian Academy of Sciences

142290 Pushchino, Moscow Region, Russian

Federation

elizaveta.rivkina@gmail.com

\section{Neslihan Tas}

Lawrence Berkeley National Laboratory

1 Cyclotron Road, MS 70A-3317

94720 Berkeley, CA, USA

ntas@lbl.gov

\section{Dirk Wagner}

GFZ German Research Centre for Geosciences, Helmholtz Centre Potsdam

Section Geomicrobiology

Telegrafenberg

14473 Potsdam, Germany

dirk.wagner@gfz-potsdam.de

\section{Mark Waldrop}

USGS

345 Middlefield Road

94025 Menlo Park, CA, USA

mwaldrop@usgs.gov

\section{Sizhong Yang}

GFZ German Research Center for Geosciences, Helmholtz Centre Potsdam

Section Geomicrobiology

14473 Potsdam, Germany

syang@gfz-potsdam.de

AND

State Key Laboratory of Frozen Soil Engineering, Northwest Institute of Eco-Environment and Resources, Chinese Academy of Sciences Lanzhou 730000, China 